\title{
An Improvement of Just Noticeable Color Difference Estimation
}

\author{
Kuo-Cheng Liu \\ Information Educating Center \\ Taiwan Hospitality \& Tourism University, Taiwan \\ Email: \{kcliu@mail.tht.edu.tw \}
}

\begin{abstract}
In this paper, the estimation of just noticeable color difference in color images is improved by using a new spatial masking. The internal generative mechanism of human of brain theory implies that the human visual system (HVS) is sensitive to the orderly stimulus possessing structural regularity which is easily to be predicted and is insensitive to the disorderly stimulus containing structural irregularity. It is obviously that the spatial masking in color images may be overestimated in the region with orderly structures and underestimated in the region with disorderly structures. By using a simple prediction model imitating the brain works of the HVS, the structural irregularity is computed to build a new masking function that can be used to improve the estimation of just noticeable color difference for color images. The masking function is further extended to build a color visual model of estimating the visibility thresholds of color images for performance comparison. Simulation results demonstrate that the proposed method is able to obtain better performance of estimating just noticeable color difference.
\end{abstract}

\section{INTRODUCTION}

$\mathrm{T}$ HE well known properties that the human visual system (HVS) has a limited sensitivity in perceiving visual information are always utilized to represent images more efficiently. Through assessing the human visual sensitivity inherent in images, the performance of many techniques has been improved in the perceptual research community and can be found in [1]-[5].

By using different levels of just noticeable color difference (JNCD), a simplified visual model introduced in [4] for estimating the perceptual redundancy for each color pixel as the visibility threshold of color difference was proposed to modify the coding efficiency of two existing image coders. In [6], Zhu et al. proposed a perceptual based no-reference objective image quality metric by integrating perceptually weighted local noise into a probability summation model. Unlike existing objective metrics, the proposed no-reference metric is able to predict the relative amount of noise perceived in images with different content, without a reference. Hsieh et al. [7] presented a copyright identification scheme for color images that takes advantage of the complementary nature of watermarking and fingerprinting was proposed to embed the watermark into the less sensitive $\mathrm{R}$ and $\mathrm{B}$ channels of the host image in the RGB color space. To gain high performance in color image processing, the properties of the human visual perception of color stimuli must be well utilized.

In this paper, the concept indicating that the brain works actively predict the input scenes and avoid the residual uncertainty/disorder is utilized to improve spatial masking for color images. Based on a simple prediction model, the luminance dominated structural irregularity is taken into account to explore a more strict spatial masking function in color images, while only background non-uniformity and texture content are used in the prior works. To avoid underestimating or overestimating the spatial masking effect in the region with structural uncertainty, the nonlinear additivity model is adopted to build a new masking function. By using this function, the visibility thresholds of color images are estimated for performance comparison under a fair viewing test.

\section{STRUCTURE-BASED ADJUSTMENT FOR SPATIAL MASKING}

In [4], the spatial masking effect considering the local color image context is exploited to calculate variable just noticeable color difference (JNCD) or variable JNCD (VJNCD) of each color pixel in the uniform CIELAB color space. That is,

$$
V J N C D_{p}=J N C D_{L a b} \cdot s_{c}\left(a_{p}, b_{p}\right) \cdot s_{L}\left(E\left(L_{p}\right), \Delta L_{p}\right)
$$

where $s_{c}\left(a_{p}, b_{p}\right)$ is a weighting function used by the CIE94 color difference equation for adjusting the dimension of the ellipsoid along the chroma a, b axis, $s_{L}\left(E\left(L_{p}\right), \Delta L_{p}\right)$ is texture masking adjustment primarily induced by average 
background luminance $E\left(L_{p}\right)$ and luminance gradient $\Delta L_{p}$ for pixel $p$ of the color image, and $J N C D_{L a b}$ is the basic visibility threshold of color difference in the CIELAB space. The basic threshold has been found around 2.3[10]. By using the colors on the surface of the VJNCD sphere, the perceptual redundancy inherent in each color pixel in color images can be estimated. According to the principle recently introduced in [8], the input scene information received by human eyes is not fully processed by the HVS and some information with structural irregularities is avoided and hard to be predicted. Herein, the structure-based adjustment, $s_{U}\left(L R_{p}\right)$, caused by considering the amount of luminance residual, $L R_{p}$, is proposed and incorporated to improve the estimation of variable just noticeable color difference. In this paper, both the structure-based adjustment and the texture masking adjustment are adequately combined to design a new spatial masking function $\Lambda(p)$ for modifying Eq. (1). For simplicity, only the luminance dominated structural irregularity inherent in the color image is investigated, while considering the fact that the human eye is more sensitive to luminance than to chrominance.

Based on the concept of internal generative mechanism of human of brain theory, the structural irregularity of an image is from the uncertain information which is hard to be predicted for the HVS. We reasonably regard the uncertain information of the image as the residual part between the image and its prediction part [9]. An computational prediction autoregressive (AR) model for the luminance component of the color image is therefore exploited and given by

$$
L_{p}^{\prime}=\sum_{p_{i} \in \Re} k_{i} L_{p_{i}}+v_{p}
$$

where $L_{p}^{\prime}$ is the predicted luminance value of pixel $p, p_{i}$ the $i$-th neighboring pixel in the surrounding region $\mathfrak{R}=\left\{p_{1}, p_{2}, \ldots, p_{N}\right\}$ and $\left\{k_{i}\right\}$ the model coefficients which are determined by minimizing the variance of the white noise $\left\{v_{p}\right\}$. The residual part is then computed as the uncertain information to construct the relation between structure-based adjustment and structural irregularity.

\section{VERIFICATION OF THE IMPROVED VARIABLE JUST NOTICEABLE COLOR DIFFERENCE}

The performance of estimating the improved variable just noticeable color difference is verified by incorporating Eq. (1) into Chou's model [4] to compare the accuracy of estimating the visibility thresholds of color images. For a particular color pixel, the perceptual redundancy is quantitatively measured by analyzing the loci of colors which are perceptually indistinguishable from this color. In [9], the loci form a sphere centralized at this color's coordinate with the radius of VJNCD in the uniform CIELAB color space and used to compute the visibility thresholds of color pixels in color images. The improved VJNCD of the color pixel $p$ within a complex image is then redefined as

$$
V J N C D_{p}^{\prime}=J N C D_{L a b} \cdot s_{c}\left(a_{p}, b_{p}\right) \cdot \Lambda(p)
$$

The procedure for estimating the perceptual redundancy of a pixel in an arbitrary color space is firstly to transform the color pixel to the CIELAB space. By using Eq. (1) that utilizes the improved spatial masking function in this paper, the corresponding improved VJNCD threshold is obtained. Under the perceptually conservative restriction controlled by the luminance, some critical colors on the surface of the improved $V J N C D$ sphere are selected to transform back to the target color space. Finally, an approximate rectangular subspace is obtained to quantify the perceptual redundancy and the visibility thresholds of the color pixel for each color channel are calculated.

The verification of the improved variable just noticeable color difference for color images is inspected by comparing the accuracy of estimating the visibility thresholds of color images in each color component. Herein, a subjective test is conducted to inspect if the estimated visibility thresholds is consistent with the HVS. Suppose a test image is represented in the $\mathrm{YCbCr}$ color space, the visibility threshold for each color pixel in each color component of the color image is randomly added to or subtracted from the corresponding color pixel. The accuracy of estimating the visibility thresholds is better if the PSNR of the contaminated image is lower, while the visual quality of the contaminated image has nearly the same as the original image under the specified viewing condition.

\section{Simulation RESUlTS}

In the viewing test, the original color image and its noise contaminated image are randomly placed side by side on the monitor. The test is carried out in the dark room when the subject observes the image pair on the monitor at a viewing distance of six times the image's height. In the simulation, a variety of standard test color images are used. The color pixels are represented in $\mathrm{YCbCr}$ format. 16 subjects who have normal eyesight or had been corrected to be normal take part in test. 


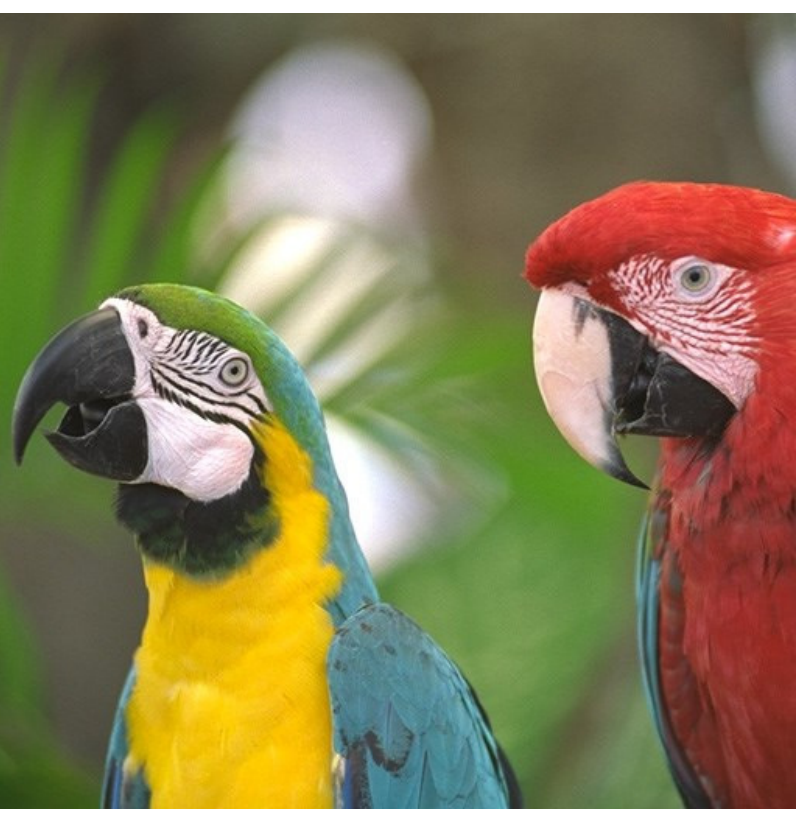

(a)

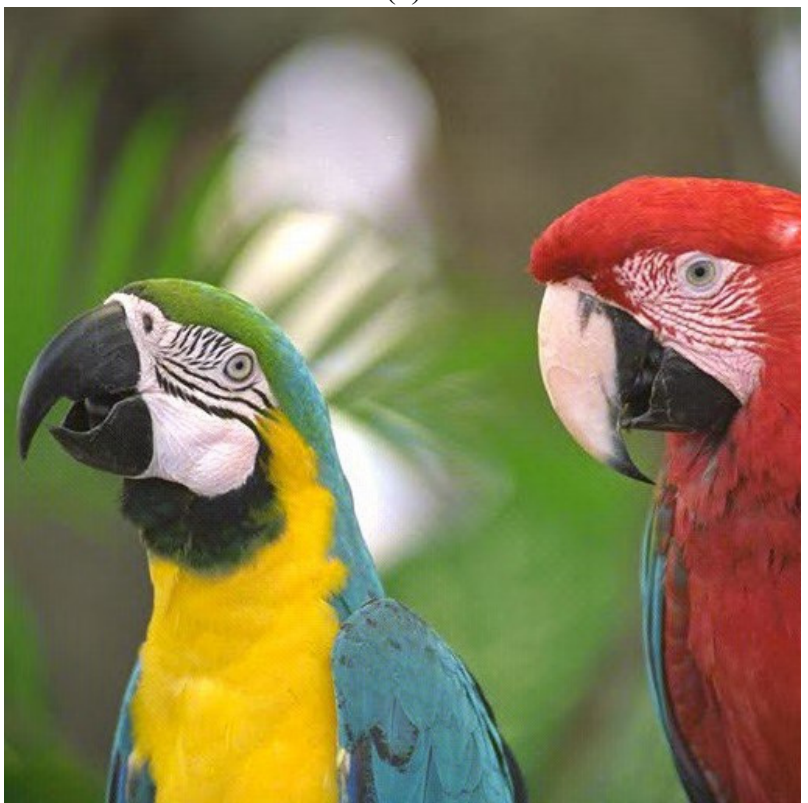

(b)

Fig. 1. (a) Original color image of "Zuerst" and (b) its noise contaminated image (PSNR=34.95B)

In Fig. 1, the original color image of "Zuerst" (Fig. 1a) and its noise contaminated version (Fig. 1b)of $\mathrm{PSNR}=34.95 \mathrm{~dB}$ by the associated visibility thresholds in three color components are shown, while the two images are perceptually indistinguishable from each other under the viewing condition mentioned above. To achieve a fair comparison for verifying the improvement of the existing variable just noticeable color difference, the simulation results are compared with the color visual model proposed in [4]. The PSNR of the noise contaminated color "Zuerst" image by the method presented in [4] is $36.87 \mathrm{~dB}$ at nearly

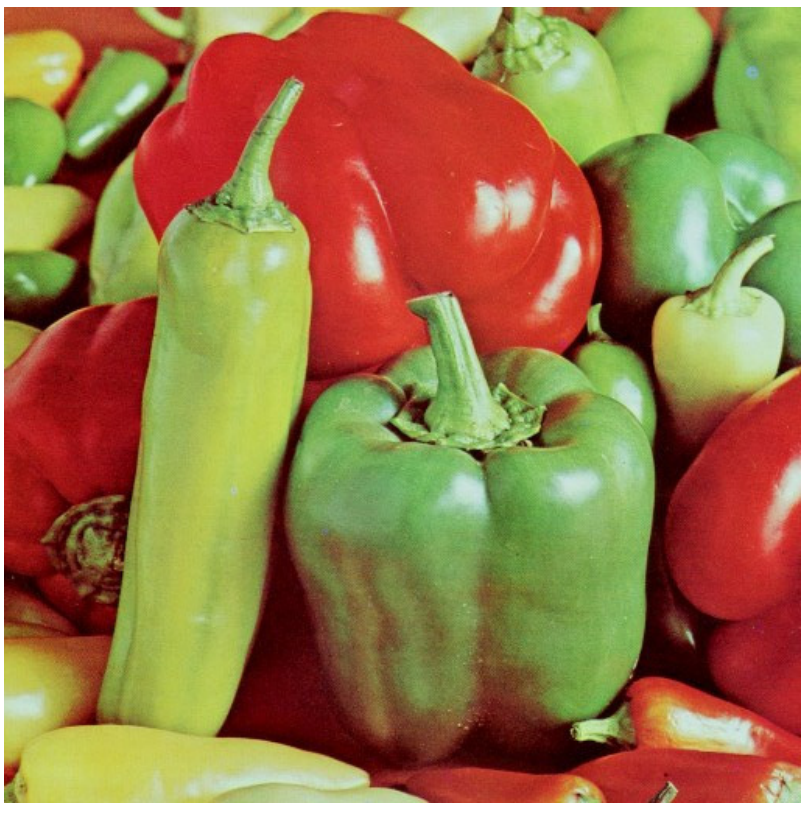

(a)

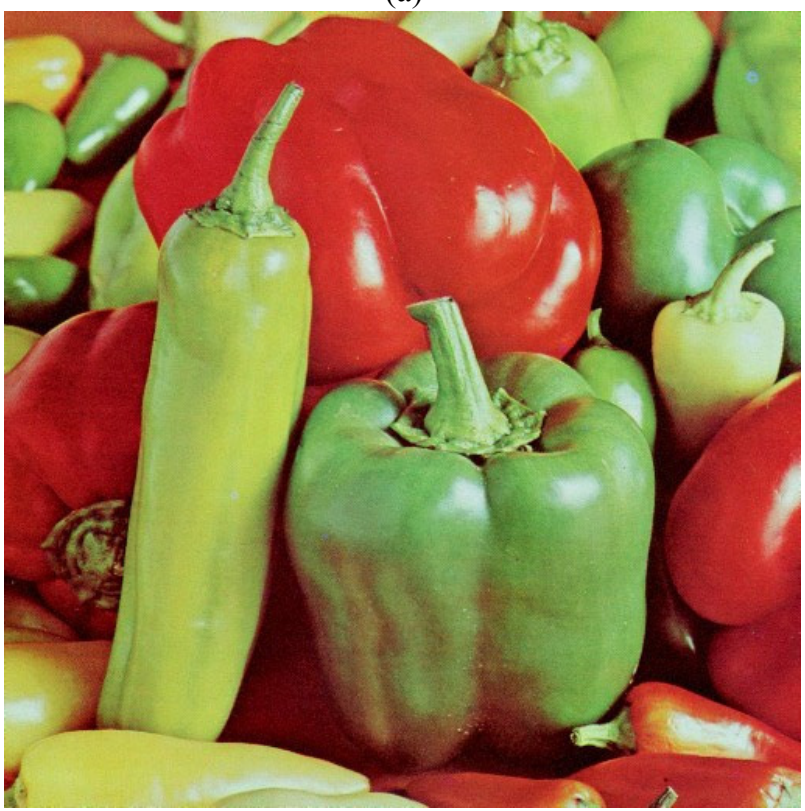

(b)

Fig. 2. (a) Original color image of "Pepper" and (b) its noise contaminated image $(\mathrm{PSNR}=33.20 \mathrm{~dB})$

the same visual quality. Fig. 2 shows the experimental results for "Pepper" color image. It is shown that the improved spatial masking estimation based on the uncertain information indeed achieve larger noise concealment in the regions with structural irregularity, while the visual quality of the noise contaminated image has nearly the same as the original image under the specified viewing condition. Under the same perceptually indistinguishable visual quality for 12 standard test color images, the average PSNR of the noise contaminated color images by the proposed method is $1.4 \mathrm{~dB}$ lower than that by the Chou's method. The 
proposed spatial masking adjustment successfully shows better performance.

\section{Conclusions}

In this paper, the estimation of just noticeable color difference in color images is improved by using a new spatial masking. By using the brain works of the human visual perception which is sensitive to the orderly stimulus that is easily predicted and is insensitive to the disorderly stimulus, the estimation is incorporated with a simple prediction model to effectively obtain a new spatial masking function. We use the function to improve the just noticeable color difference for computing the visibility thresholds of color images for performance comparison. With the new spatial masking function, the proposed spatial masking adjustment successfully shows better performance than the existing masking method.

\section{ACKNOWLEDGMENT}

The work was supported by the Ministry of Science and Technology, Taiwan (R.O.C.), under contract MOST 1042221-E-278-001, and Image \& Video Processing Laboratory of Department of Computer Science and Information Engineering, National Dong-Hwa University, Taiwan.

\section{REFERENCES}

[1] H. Tian, Y. Fang, Y. Zhao, W. Lin, R. Ni, and Z. Zhu, "Salient Region Detection by Fusing Bottom-Up and Top-Down Features Extracted From a Single Image”, IEEE Trans. Image Process., vol. 23, no. 10, pp. 4389-4398, 2014.

[2] H. R. Sheikh and A. C. Bovik, "Image information and visual quality," IEEE Trans. Image Process., vol. 15, no. 2, pp. 430-444, Feb. 2006.

[3] I. Höntsch and L. J. Karam, "Locally adaptive perceptual image coding,” IEEE Trans. Image Processing, vol. 9, no. 9, pp. 1472-1483, Sep. 2000.

[4] C. H. Chou and K. C. Liu, "Colour image compression based on the measure of just noticeable colour difference," IET Image Processing, vol. 2, no. 6, pp.304-322, Dec. 2008.

[5] P. B. Nguyen, A. Beghdadi, M. Luong, "Perceptual watermarking using a new JND model," Signal Processing: Image Communication, vol. 28, no. 10, pp. 1506-1525, Nov. 2013.

[6] T. Zhu and L. Karam, "A no-reference objective image quality metric based on perceptually weighted local noise," EURASIP Journal on Image and Video Processing, vol. 1, no. 5, pp. 1 - 10, 2014.

[7] S.-L. Hsieh, C.-C. Chen, and W.-S. Shen, "Combining Digital Watermarking and Fingerprinting Techniques to Identify Copyrights for Color Images," Scientific World Journal, Jan. 2014 http://dx.doi.org/10.1155/2014/454867.

[8] K. Friston, "The free-energy principle: A unified brain theory?" Nat. Rev. Neurosci., vol. 11, no. 2, pp. 127-138, Feb. 2010.

[9] J. Wu, G. Shi, W. Lin, A. Liu, and F. Qi, "Just Noticeable Difference Estimation For Images with Free-Energy Principle”, IEEE Trans. Multimedia, vol. 15, no. 7, pp. 1705-1710, Nov. 2013.

[10] M. Mahy, L. Van Eyckden, and A. Oosterlinck, "Evaluation of uniform color spaces developed after the adoption of CIELAB and CIELUV,” Color Res. Appl., vol. 19, no. 2, pp. 105-121, 1994. 